\title{
Mappare la distruzione Locative media e narrazione del sisma ad Amatrice
}

\author{
Marta Milia \\ Università degli Studi di Torino \\ miliamarta@tiscali.it \\ $@$ miliamarta1
}

\begin{abstract}
Il contributo intende analizzare il rapporto che intercorre tra la pratica di geolocalizzazione, attraverso dispositivi mobili e social media, e la gestione di avvenimenti particolarmente traumatici per una collettività. Lo studio riguarda il terremoto che ha colpito il centro Italia nell'agosto 2016 e in particolare il centro di Amatrice, con un focus sulla narrazione e la localizzazione delle attività quotidiane degli individui. La riflessione sul rapporto tra Locative Media e narrazione della quotidianità, del vissuto attraverso la collocazione spaziale, della distruzione non solo dei luoghi fisici ma anche metaforici, si articola attraverso lo studio e il monitoraggio di queste "mappature del senso" sui social media, ben individuabili e distinguibili attraverso marcatori propri della loro diffusione, come ad esempio gli hashtag. È un processo narrativo individuale che si concretizza e diventa "fruibile" da altri individui proprio attraverso i cosiddetti locative media, i quali permettono la condivisione non solo di una posizione geografica corredata da immagini, frasi, video, ma anche la condivisione di un vissuto che da personale e intimo si evolve diventando narrazione pubblica, visibile, analizzabile e condivisibile da altri utenti.
\end{abstract}

\section{Parole chiave}

Locative Media, Social Media, Terremoto, Narrazioni Social, Geosemiotica

\begin{abstract}
Mapping the destruction. Locative Media and the narration of the earthquake in Amatrice - This contribution intends to analyze the relationship between the practice of geolocation, through mobile devices and social media, and the management of events having a particularly traumatic impact on a community. The study concerns the earthquake that struck central Italy on August 2016 and in particular the town of Amatrice, with a focus on the narration and localization of the daily activities of individuals. The reflection on the relationship between locative Media and the narration of everyday life, of the experience through the space-based location and of the destruction not only of physical but also of metaphorical places, is articulated through the study and monitoring of such "sense mappings" on social media. They are well recognizable and distinguishable through markers proper of their diffusion, such as the hashtags. Such individual narrative process becomes "usable" by other individuals through the so-called Locative Media, which allow to share not only a geographical position accompanied by images, phrases, videos, but also the personal and intimate experiences which evolve into visible, analyzable and public narratives, that can be shared by other users.
\end{abstract}

\section{Key Words}

Locative Media, Social Media, Earthquake, Social Narrations, Geosemiotics 


\section{Ocula ${ }^{21}$}

Geosemiotica: dai locative media, alle immagini diffuse, ai big e small data

Marta Milia | Mappare la distruzione | DOI:10.12977/ocula2019-19

\section{Sommario}

1. Locative media: introduzione

1.2. Tipologie e aspetti discorsivi

2.Geolocalizzare il vissuto: narrazione geografica di un terremoto

2.1. Gli strumenti della georeferenziazione

3. Locative media, utenti e la diffusione di senso

Bibliografia 


\section{Ocula ${ }^{21}$}

Geosemiotica: dai locative media, alle immagini diffuse, ai big e small data

Marta Milia I Mappare la distruzione I DOI:10.12977/ocula2019-19

\section{Locative media: introduzione}

La riflessione e lo studio sugli aspetti comunicativi e la nascita di nuovi codici relazionali attraverso i social media, diventa sempre più complesso e articolato e si evolve di pari passo con lo sviluppo di nuove tecnologie applicabili ai più diffusi device oggi in nostro possesso: gli smartphone in particolare, risultano essere lo strumento con il quale maggiormente avviene la diffusione del "senso" attraverso i social media più diffusi e le applicazioni più disparate. Condividere immagini, informazioni, musica e luoghi attraverso il proprio smartphone rappresenta una delle azioni più identificative e probanti della nostra esistenza nella realtà digitale, non solo: la presenza dell'individuo sui social media non ha valore soltanto nella realtà digitale ormai, ma è un elemento valoriale riconosciuto anche nella realtà "reale" dove tutto avviene concretamente. La presenza attiva e dinamica di un individuo e la sua agentività nel mondo social è percepibile e valutabile dunque anche al di fuori di esso. Nel mondo degli oggetti concreti.

I luoghi, la loro valenza simbolica e la loro capacità di raccontare sono al centro di un massiccio sviluppo di pratiche e sistemi di diffusione di tendenze correlate dunque alla dimensione locative di numerose attività quotidiane, eventi, fatti di cronaca e persino eventi tragici come il terremoto del Centro Italia. Questo processo è stato già da qualche tempo potenziato dall'utilizzo di dispositivi in grado di localizzare, georeferenziare e inserire in un contesto spaziale l'individuo e le sue attività: il rapporto con il luogo nel quale si trova, le sue attività e le connessioni, magari in condivisione con altri individui presenti nel medesimo spazio, permette di sviluppare una diffusione di narrazioni su scala massiccia e di analizzarne il flusso di dati sui social media attraverso applicativi specifici, in grado di tracciare una vera e propria "mappa del senso" relativa al luogo prescelto.

Il termine locative media fa la sua prima apparizione nel 2003, e viene utilizzato dall'artista Karlis Kalnins per definire quei processi di catalogazione degli spazi fisici a livello digitale attraverso l'utilizzo di tecnologie quali il Wi-Fi o il GPS: l'oggetto di valore risiederebbe quindi nel carattere situato e georeferenziato dell'interazione.

Questi sistemi hanno consentito di tracciare una mappa dei movimenti degli utenti all'interno di una dimensione spaziale definita, come ad esempio la città, condividendo informazioni, realizzando uno scambio di contenuti sia nella realtà spaziale che nello spazio virtuale e innescando così un processo di stratificazione ibrido che coinvolge spazi fisici e dati elettronici. Bertone (2013) associa a questo termine, inoltre,

quelle particolari esperienze che utilizzano le tecnologie di posizionamento con scopi marcatamente sociali, culturali e pubblici, per favorire l'emergere di nuove esperienze situate e nuove relazioni tra persone, tecnologie e spazi fisici. 


\section{Ocula ${ }^{21}$}

Geosemiotica: dai locative media, alle immagini diffuse, ai big e small data

Marta Milia I Mappare la distruzione I DOI:10.12977/ocula2019-19

Secondo questa affermazione, quindi, con l'espressione Locative Media non ci si riferisce soltanto a oggetti fisici in grado di svolgere un determinato compito, ma funge da "contenitore" per un insieme eterogeneo di strumenti ed esperienze etichettabili sotto il medesimo significante.

Le interfacce grafiche con le quali si rapporta l'utente hanno quindi il potere di far accedere l'individuo a uno spazio narrativo virtuale e contemporaneamente tradurre le sue esperienze fisiche concrete in narrazioni digitali in perpetua connessione tra loro (Graham, Marvin,2002).

\subsection{Tipologie e aspetti discorsivi}

I locative media in quanto tali hanno avuto origine a partire da progetti sviluppati da Google e gli applicativi my Location e Google Latitude, inclusi nella app per dispositivi mobili Google Maps, utili per localizzare ciò che circonda l'utente e la sua collocazione nello spazio ("Casa", "Luoghi vicini a te" e così via) e la posizione di amici e membri della community. Da citare anche Fire Eagle, chiuso nel 2013, il servizio creato da Yahoo! per la geolocalizzazione degli utenti. A segnare una svolta nel sistema locative è stato Foursquare, fondato nel 2009: non solo è possibile segnalare la propria posizione ad altri utenti, attraverso un dispositivo mobile con l'app dedicata, ma gli utenti tramite il check-in, possono registrarsi in locali, negozi, musei e numerosi altri esercizi commerciale o di pubblico interesse. Va inoltre considerato l'effetto social marketing di Foursquare, per mezzo del quale le aziende possono promuovere il proprio brand, offrire sconti e agevolazioni agli utenti più assidui. L'aspetto ludico di questo locative media (anche considerato un vero e proprio gioco sociale partecipativo), è individuabile nel traffico dei check e dei tip, ovvero feedback sui luoghi visitati. Più se ne accumulano, più badge, "medaglie" riguardanti la popolarità e l'influenza di un utente si guadagnano. Inoltre, i contenuti dell'applicazione sono sincronizzabili con Twitter e Facebook, aumentando quindi esponenzialmente i contatti e ampliando le connessioni. L'obiettivo degli utenti è quello di diventare "sindaco" del luogo dove maggiormente l'utente ha fatto i check-in in minor tempo. Nel tempo il sistema si è evoluto e Foursquare ha sviluppato una seconda app, Swarm, dedicata esclusivamente al social meeting mentre Foursquare può essere utilizzata esclusivamente per visitare e recensire i luoghi di interesse visitati.

Per quanto riguarda i social media maggiormente utilizzati dagli utenti, Facebook ha sviluppato ormai da qualche anno Nearby Friends (amici nelle vicinanze) che avvisa l'utente se ci sono altri amici nella zona e un particolare servizio di Safety Check (attivabile solo dagli amministratori del social network) in presenza di situazioni di pericolo collettivo come atti terroristici e catastrofi naturali (come nel caso del terremoto in Italia), fino alle funzionalità location aware (localizzazione consapevole) integrate. Instagram e Twitter, che permettono agli utenti di attivare la posizione sulla barra principale e che poi sarà visibile in tutti i tweet successivi.

La componente georeferenziale risulta certo imprescindibile per la comunicazione sui social media e la creazione di nuove reti sociali, grazie alle quali si creano ulteriori gruppi che rafforzano l'enunciazione intorno a topic specifi- 


\section{Dcula ${ }^{21}$}

Geosemiotica: dai locative media, alle immagini diffuse, ai big e small data

Marta Milia | Mappare la distruzione I DOI:10.12977/ocula2019-19

ci su luoghi di particolare interesse e su avvenimenti in grado di suscitare dal punto di vista emotivo un'attenzione a livello globale.

Il processo che regola la narrazione virtuale e il luogo fisico oggetto della stessa, prevedono un piano di condivisione dei contenuti immessi in rete all'interno del quale l'utente narratore si espone, mette in evidenza la dimensione patemica del vissuto legata alla posizione geografica, ossia lo spazio prescelto per la condivisione in un particolare momento e con un particolare stato d'animo.

Condivisione, immediatezza e coinvolgimento sono quindi alcuni degli aspetti più importanti nell'uso dei Locative Media quali veicoli dinamici di narrazioni attraverso gli spazi e contribuiscono a sviluppare connessioni sociali sempre più basate su un approccio digitale votato alla narrazione delle esperienze su luoghi concreti.

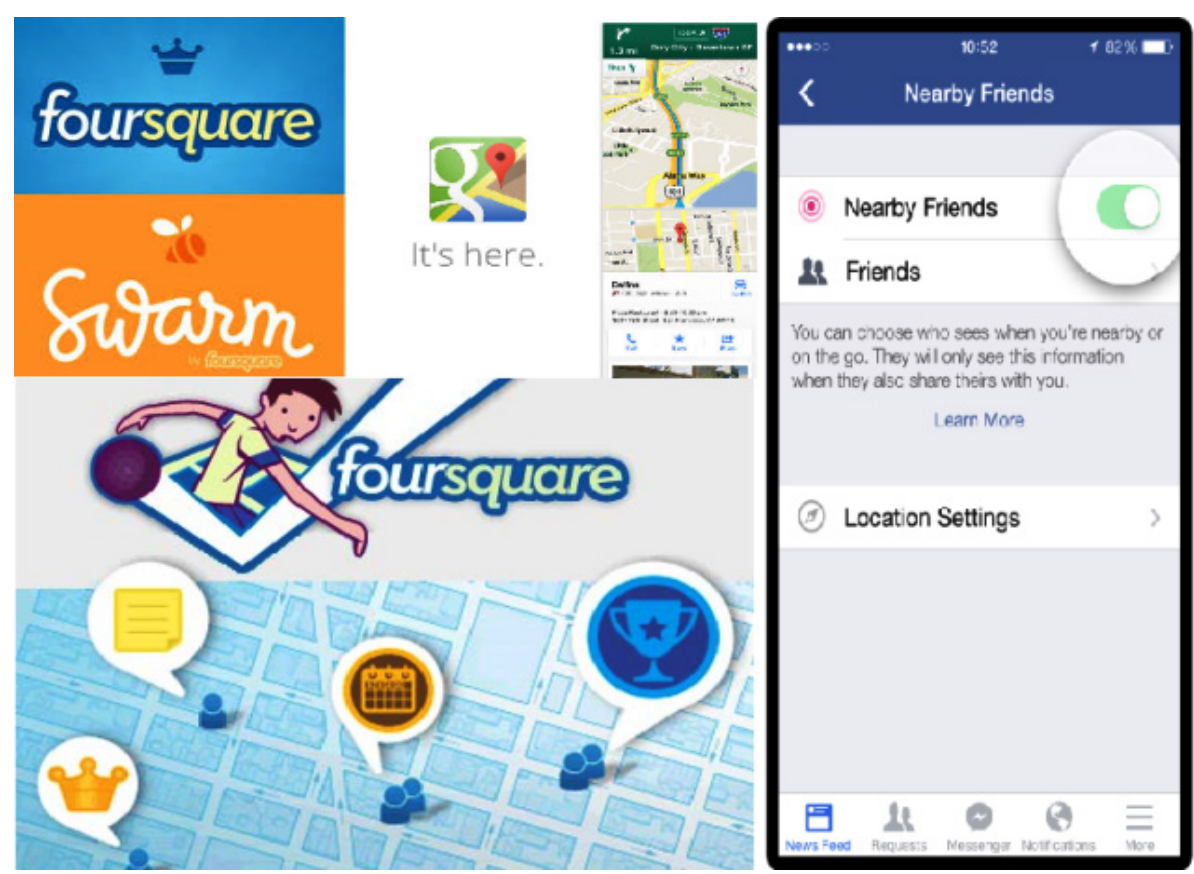

Figura 1. Locative media.

\section{Geolocalizzare il vissuto: narrazione geografica di un terremoto}

Fondamentale dunque l'individuazione da parte dell'utente di quel luogo geografico deputato alla creazione e diffusione di senso; Vellega (2003) afferma che "La geografia è stata considerata come la descrizione metaforica del territorio", in grado quindi di rappresentare la realtà basandosi su elementi convenzionali, riconosciuti a livello collettivo: dei simboli creati dalla comunità e da essa assimilati.

Da un punto di vista semiotico, dunque, l'individuo come tale o come membro di un gruppo attribuisce ai simboli presenti negli spazi geografici un valore, li dota di un significato ulteriore, oltre a quello riconosciuto collettivamen- 


\section{Deula ${ }^{21}$}

Geosemiotica: dai locative media, alle immagini diffuse, ai big e small data

Marta Milia I Mappare la distruzione I DOI:10.12977/ocula2019-19

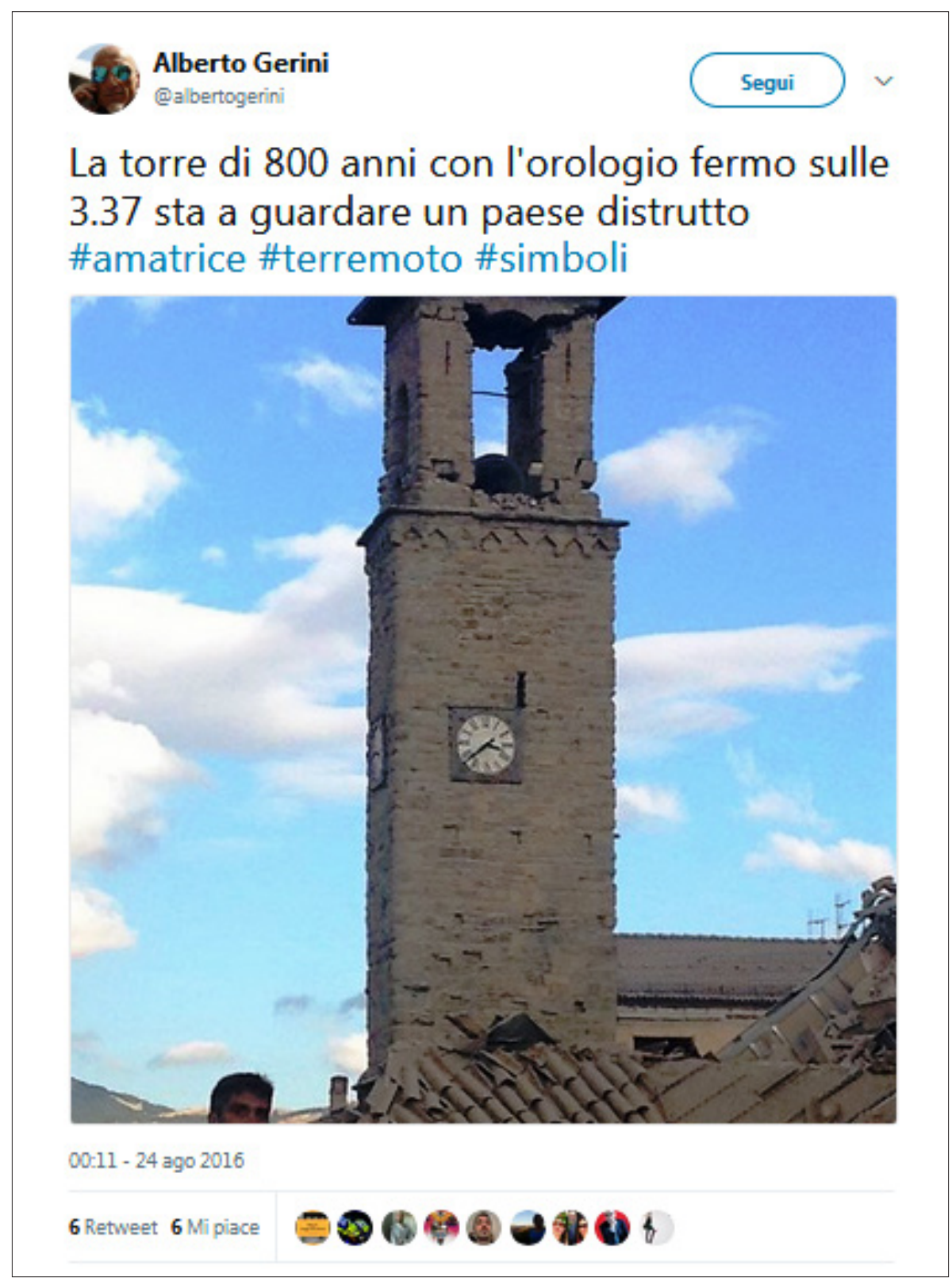

Figura 2. Simboli della distruzione.

te: il simbolo (che, come vedremo, riguarda i luoghi colpiti dal sisma) subisce un'ulteriore trasformazione da parte dell'individuo-utente che attribuisce ad esso una valenza del tutto personale, privata, la quale scaturisce da ricordi, sensazioni e situazioni pregresse. Lo spazio è manipolato ulteriormente da un soggetto agente che si dota delle competenze necessarie per donargli un valore specifico: referenziarlo con un nome e dei confini non necessariamente aderenti a quelli riconosciuti universalmente e che, per mezzo dei locative media, viene interpretato e recepito da altri utenti partecipi del medesimo interesse in quello spazio. 


\section{Ocula ${ }^{21}$}

Geosemiotica: dai locative media, alle immagini diffuse, ai big e small data

Marta Milia I Mappare la distruzione I DOI:10.12977/ocula2019-19

\subsection{Gli strumenti della georeferenziazione}

Ma quali sono gli strumenti e le competenze delle quali si dota l'individuo-utente nel processo di georeferenziazione delle esperienze, in particolare di avvenimenti traumatici e disastrosi?

Lo smartphone, in prima istanza, è il device maggiormente deputato alle attività di geolocalizzazione: le sue proprietà e l'uso che l'utente fa di questo dispositivo, non rappresenta una semplice estensione delle pregresse pratiche proprie di Internet. Si possono consultare i Social Media e lavorare con la rete come con qualunque computer; tuttavia, molte delle applicazioni presenti sui dispositivi mobile permettono la localizzazione fisica dell'utente nello spazio.

Lo smartphone è infatti dotato degli applicativi necessari alla collocazione dell'individuo nel territorio, alla condivisione di tale posizione e alla narrazione dell'azione nel momento in cui si svolge grazie alle foto che può scattare e ai video che può girare. Lo smartphone è dunque lo strumento attraverso il quale il soggetto "manipola" il luogo predetto, o meglio, lo spazio entro il quale agisce. L'utente, forte delle esperienze e del vissuto che lo lega a quel luogo, se ne appropria, lo rende altro da ciò per il quale era stato creato attraverso una pratica tanto "quotidiana" quanto creativa come quella della geolocalizzazione.

I dispositivi mobile sono fondamentali per la diffusione della narrazione e affinché il processo enunciativo si compia: attraverso la lente della fotocamera e poi sullo schermo dello smartphone, l'utente immortala e cattura l'immagine del momento e del luogo nel quale si compie l'azione: questo istante cruciale viene poi, sempre attraverso il device, condiviso e reso fruibile. L'immagine sotto lo schermo, malgrado si riferisca a un istante ormai passato e a un luogo lontano e suscettibile di modiche, dove le persone e le cose mutano, conserva quel valore di "sospensione" temporale grazie al processo di condivisione e percepito come contemporaneo dagli altri utenti che la visualizzano.

Nel caso specifico del sisma che ha colpito il centro Italia il 24 agosto 2016, sono molte le immagini e i luoghi immortalati e poi condivisi sui social media: centinaia di migliaia di tweet hanno contribuito a costruire la narrazione di un disastro naturale e umano fra i più drammatici della storia italiana contemporanea. Georeferenziare gli istanti immediatamente successivi alle scosse, etichettare i luoghi della distruzione attraverso gli strumenti propri dell'enunciazione digitale, ha permesso agli utenti della rete di prendere coscienza in brevissimo tempo della portata dell'evento e delle sue terribili conseguenze: non solo con l'attivazione della geolocalizzazione, ma anche per mezzo della marcatura dei luoghi delle tragedie attraverso hashtag dedicati quali:

\author{
\#Amatrice \\ \#Accumoli \\ \#PrayforLazio \\ \#PrayforItaly \\ \#TerremotoItalia \\ \#Norcia
}




\section{Ocula ${ }^{21}$}

Geosemiotica: dai locative media, alle immagini diffuse, ai big e small data

Marta Milia | Mappare la distruzione I DOI:10.12977/ocula2019-19

Questi esempi fungono infatti da marcatori geografici per l'immediato riconoscimento della dimensione spaziale della tragedia.

Ulteriori tracce dell'enunciazione geografica del terremoto sono individuabili nei post pubblicati da chi ha assistito in prima persona allo spettacolo della distruzione nelle ore successive al sisma: l'enunciazione acquista quel valore di sospensione temporale con i tweet corredati dalle immagini delle macerie, simbolo inequivocabile della violenza delle scosse che hanno sconvolto Amatrice.

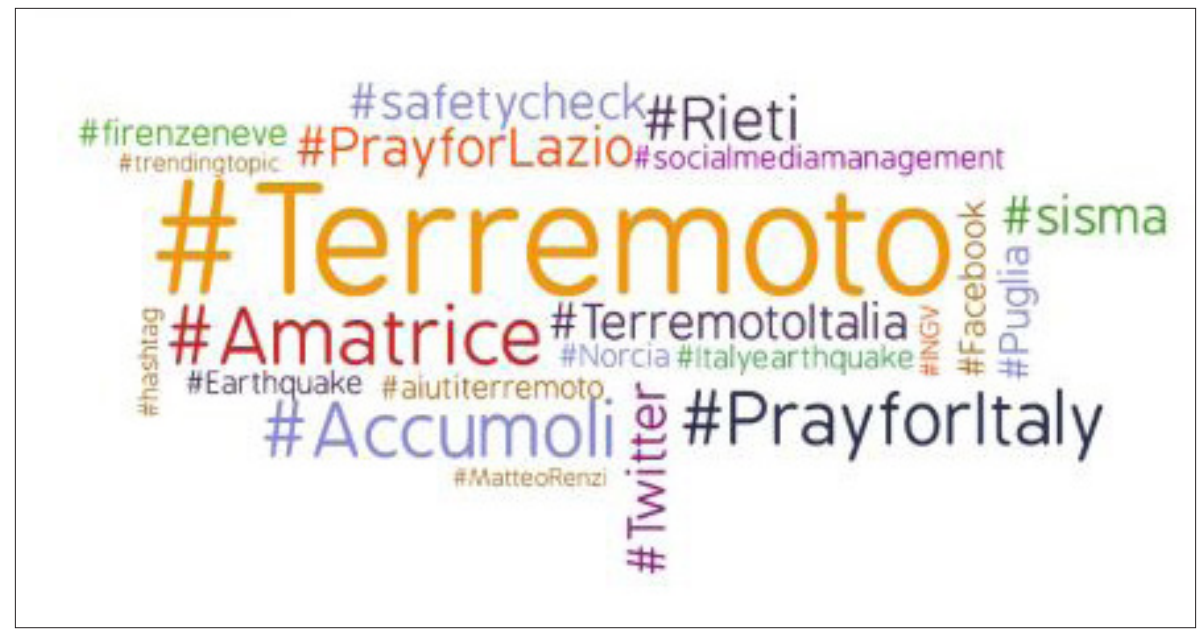

Figura 3. Tag Cloud terremoto.

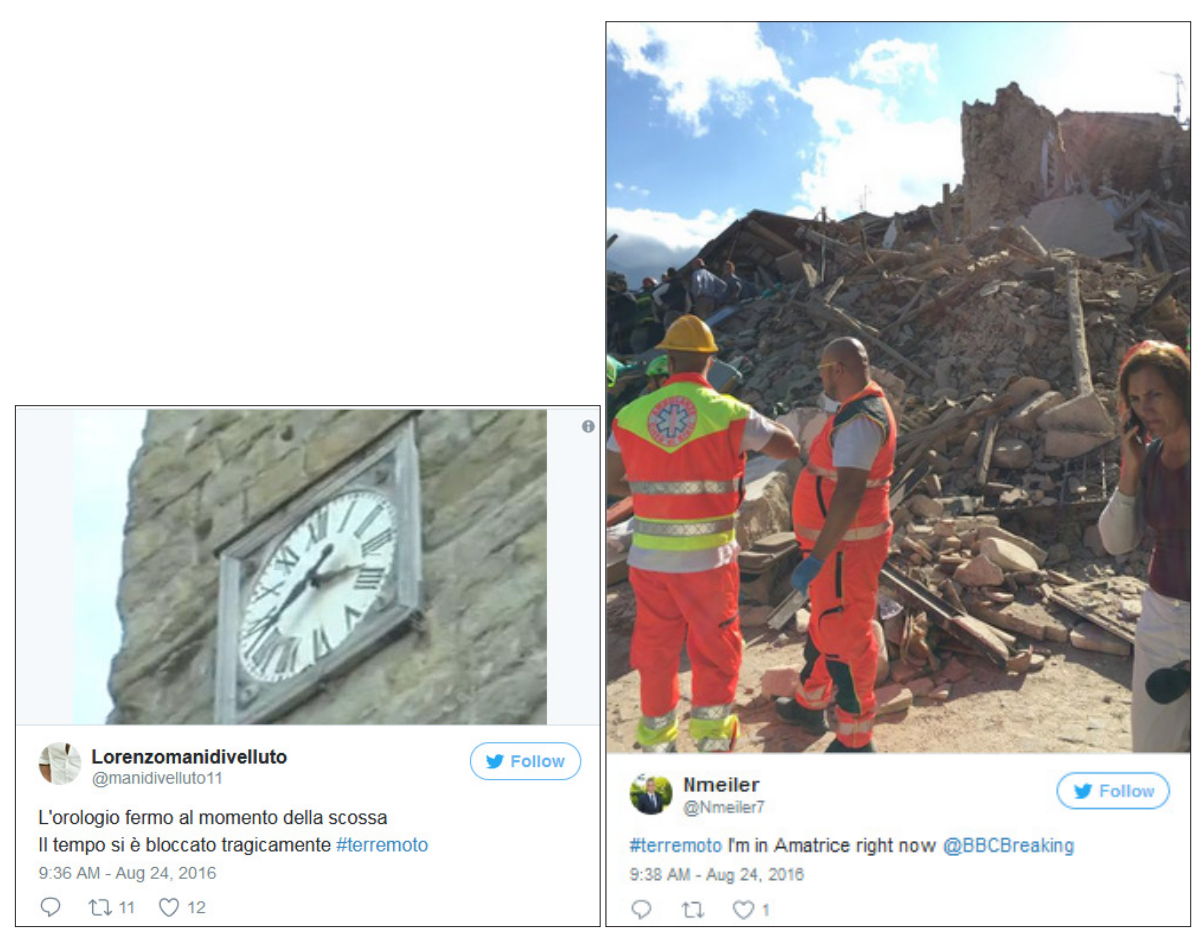

Figura 4. Tweet della distruzioni. 


\section{Dcula ${ }^{21}$}

Geosemiotica: dai locative media, alle immagini diffuse, ai big e small data

Marta Milia I Mappare la distruzione I DOI:10.12977/ocula2019-19

In entrambi i tweet è possibile scorgere sia i marcatori temporali che quelli geografici della narrazione: ora giorno e luogo. Le foto all'interno del tweet, tuttavia, raccontano qualcosa in più. Le immagini immortalano le macerie, cumuli di detriti che fino a poche ore prima erano abitazioni. I tweet decodificano solo in parte il contenuto delle foto, ma definiscono con precisione il luogo dell'accaduto come a re-identificare un simbolo ormai sfigurato e irriconoscibile.

Stefano Sbrulli| @sbrullis

\#terremoto Piu' il sole si alza, piu' ci rendiamo conto che \#Amatrice e' distrutta!

9:38 A.M. Aug 24, 2016

Nmeiler @Nmeiler7

\#terremoto I'm in Amatrice right now @BBCBreaking

9:38 A.M. Aug 24, 2016

Il testo dei Tweet rivela la presenza del narratore, che sta dietro l'obiettivo dello smartphone e che testimonia la sua presenza ad Amatrice dopo il sisma. I marcatori temporali e spaziali, gli elementi fondamentali della narrazione dei luoghi colpiti, ci confermano quello che le foto testimoniano. Distruzione e perdita di ogni punto di riferimento spaziale in grado di connotare quella che una volta era una cittadina turistica conosciuta internazionalmente.

Le marcature spaziali digitali, dunque, diventano in questo frangente gli unici veri simboli in grado di suggerire a chi è lontano da Amatrice che quella è realmente Amatrice. Se nella figura 2 il tweet descrive e immortala uno spazio simbolo ancora decodificabile e riconoscibile come parte integrante del tessuto urbano della città, la figura 4, senza la georeferenziazione, non sarebbe in grado di descrivere e quindi confermare all'utente lontano dal disastro che effettivamente si tratta di foto scattate ad Amatrice.

L'utente presente sul luogo del disastro ricopre il ruolo di portavoce e rappresentante di una comunità che, pur essendo nel mezzo della tragedia, ne prende le distanze e racconta attraverso le coordinate geografiche e dettagli universalmente riconosciuti (nome del luogo, ora e data) la cronaca non solo della città ma del suo vissuto personale: ciò che noi vediamo è ciò che l'utente portavoce ha realmente immortalato, i suoi occhi hanno visto le macerie realmente. L'esperienza si trasforma poi implicitamente in una narrazione corale nel momento in cui l'utente del primo tweet utilizza la deissi implicita /noi/ in luogo dell'implicito /io/ nella presa di coscienza della distruzione. Il lettore utente percepisce quel /noi/ come una presenza nello spazio di più persone oltre il portavoce, un'esperienza, quindi, sebbene individuale nella condivisione in rete per mezzo dello smartphone, collettiva nella dimensione geografica reale. Le sensazioni dell'utente portavoce, presumibilmente, vengono condivise dagli altri superstiti e, con l'azione di condivisione, il tweet che rende l'esperienza del terremoto pubblica, crea dei "superstiti virtuali", ovvero ricrea la situazione di condivisione della visione del paesaggio distrutto nella realtà, sulla piattaforma social (v. fig. 5). 


\section{Dcula ${ }^{21}$}

Geosemiotica: dai locative media, alle immagini diffuse, ai big e small data

Marta Milia | Mappare la distruzione | DOI:10.12977/ocula2019-19

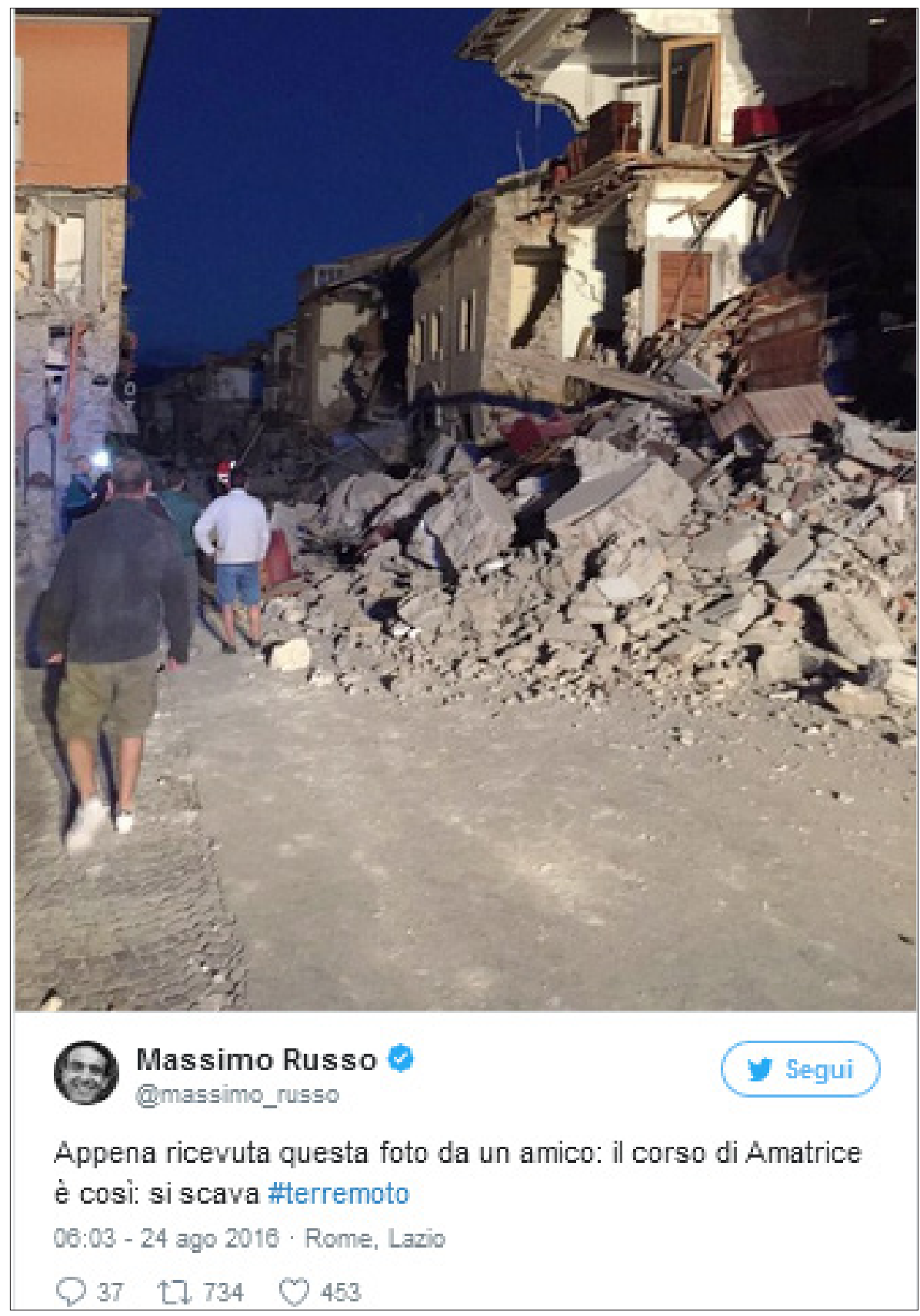

Figura 5. Terremoto Amatrice.

Le pratiche di narrazione delle geografie dei luoghi devastati dal sisma creano un effetto domino nella diffusione del senso, spingendo sempre più utenti a condividere in rete immagini e video, inviati a loro volta da amici e parenti, implicati direttamente e quindi testimoni oculari di ciò che è stato condiviso. 


\section{Ocula ${ }^{21}$}

Geosemiotica: dai locative media, alle immagini diffuse, ai big e small data

Marta Milia I Mappare la distruzione I DOI:10.12977/ocula2019-19

\section{Locative media, utenti e diffusione di senso}

Nell'affrontare l'analisi del ruolo dei locative media durante il sisma, è stato necessario soffermarsi non solo sulla funzione primaria dello strumento "aiutante" in grado di documentare in tempo reale la tragedia, ma del suo ruolo di trasmettitore di dati e informazioni sugli utenti vittime e sui luoghi colpiti. Il contributo degli utenti della rete, infatti, ha innescato un meccanismo in grado di dare vita ad altre narrazioni collaterali e correlate a quella primaria.

Un veicolo narrativo e strumento locative altamente utile è stato il sistema Safety Check attivato da Facebook pochi istanti dopo la catastrofe. Questo applicativo della piattaforma permette di notificare agli utenti geolocalizzati presenti nell'area del terremoto un messaggio per avere informazioni sul loro stato di salute.

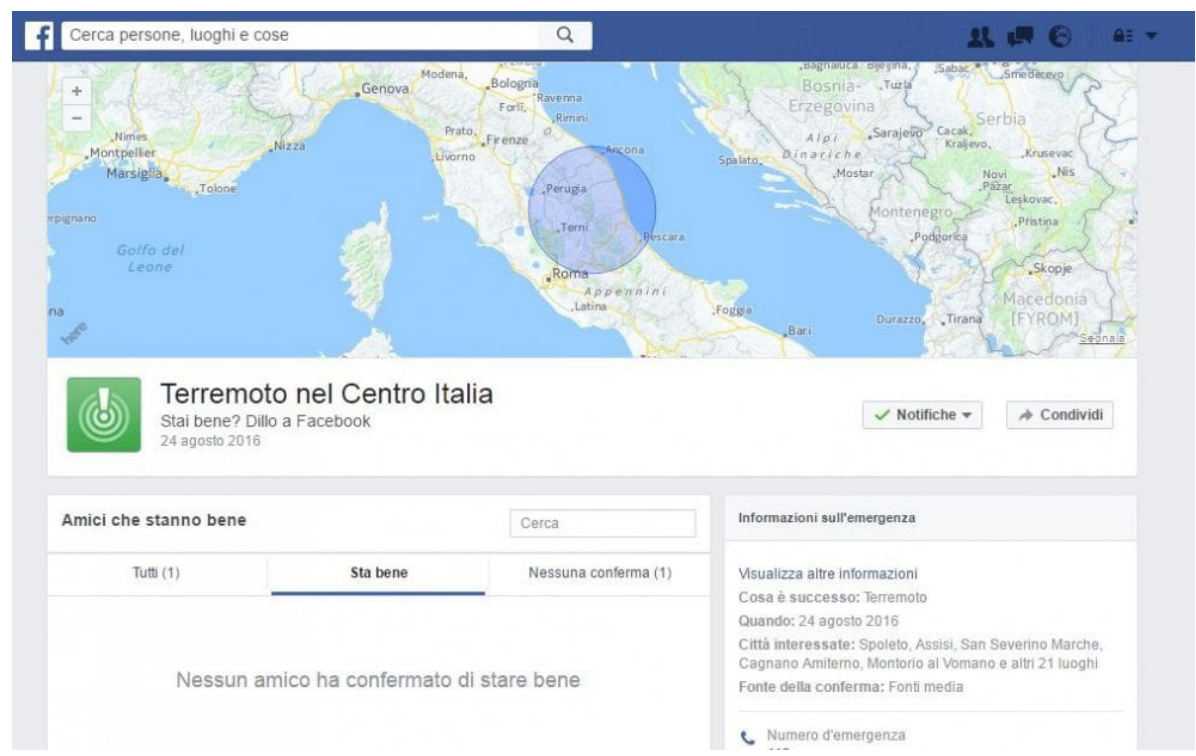

Figura 6. Pagina "Safety Check" di Facebook durante il terremoto.

Il dispositivo diventa un contenitore implicito di micro narrazioni originate dal feedback positivo e negativo degli utenti-vittime che aderiscono al patto comunicativo proposto dall'interfaccia. Le micronarrazioni vengono a loro volta consultate da utenti estranei alla vicenda ma preoccupati per i propri cari, aggiungendo così spettatori all'azione narrativa. Questo servizio, basato sulla posizione ha permesso quindi non solo di tracciare una mappa dei luoghi della tragedia, ma anche una che contenesse le geografie dell'emotività: il sollievo per la conferma di chi è sopravvissuto e lo sconforto per chi non ha aderito al patto del Safety Check. Lo smartphone, dunque, fondamentale strumento per mezzo del quale è possibile monitorare e partecipare alle narrazioni locative, alla luce degli aspetti sopra citati assume una particolare caratteristica, definita da La Cecla (1992 p. 32) con il termine di oggettualità, ovvero "la qualità di presenza di un oggetto, la sua capacità efficace di interagire con chi lo usa, il suo essere capace di trasformare l'utente". L'oggettualità dello smar- 


\section{Dcula ${ }^{21}$}

Geosemiotica: dai locative media, alle immagini diffuse, ai big e small data

Marta Milia I Mappare la distruzione I DOI:10.12977/ocula2019-19

tphone quindi rappresenta una dimensione all'interno della quale utente e oggetto si fondono delineando un unico orizzonte di senso incentrato sull'uso e la risignificazione e originando un'unica entità difficilmente scomponibile.

Il locative media per mezzo dello Smartphone-aiutante immortala dunque il qui e ora del vissuto tragico e della distruzione mentre il soggetto lo riproduce materialmente in rete: la percezione di una contemporaneità dell'evento, grazie a questa pratica, viene avvertita grazie alle tracce indelebili che la narrazione lascia in rete, anche a distanza di tempo. Le immagini shock, i contributi degli utenti, e la trasformazione dello spazio in luogo altro, distrutto e irriconoscibile, operano nell'utente una destrutturazione dei sentimenti e della percezione della realtà. L'idea veicolata dall'utente soggetto di condividere attraverso i locative media le immagini del terremoto, rappresenta la prova della reale sua presenza sul posto, una testimonianza attendibile dell'accaduto e dell'esperienza di smarrimento e disorientamento.

Il trauma viene pertanto gestito attraverso la condivisione del vissuto con altri utenti, un racconto della tragedia ripercorrendo i luoghi del disastro, del ricordo e di ciò che era e non sarà più. Lo smantellamento, insieme al paesaggio fisico di tutto ciò che era legato ad esso, attraversa l'esperienza personale dei singoli e della collettività. I simboli georeferenziati nel passaggio dal prima al dopo la tragedia, sembrano, in modo sistematico, quasi aspirare alla costruzione di un progetto di condivisione della memoria (la scuola, il campanile, la via principale).

I locative media si distinguono in questo senso come possibile strumento per lo sviluppo di un'enunciazione che mira al riconoscimento e alla consapevolezza di un disastro non solo naturale ma anche sociale, umano. Mappare i luoghi del lutto significa mappare il lutto stesso, il senso della tragedia e le sue conseguenze dirette e indirette. Ciò che scaturisce dal terremoto è purtroppo sotto gli occhi di tutti.

L'ambiente, lo spazio corrotto, racconta ancora prima dei giornalisti ciò che è realmente successo: la sua collocazione in un contesto preciso, come quello digitale, risulta in questo caso di vitale importanza per fare una stima dei danni, aggiornare sulla condizione delle vittime, riconoscere luoghi e far capire a chi non è presente lo stato reale delle cose.

Entra in gioco quindi una narrazione geografica ben più complessa e benché estremamente individualista nella sua creazione nell'implicito "sono qui, ma sto bene", diviene ben presto corale nella sua diffusione e condivisione di stati d'animo e vissuti comuni.

\section{Bibliografia}

Barreneche, Carlos

2015 "Platform specificity and the politics of location data extraction", European Journal of Cultural Studies, Sage, pp. 597-513.

Bertone, Giulia

2010 "Locative media: una perlustrazione al City Centered Festival" (San Francisco, California, USA), REM. 


\section{Dcula ${ }^{21}$}

Geosemiotica: dai locative media, alle immagini diffuse, ai big e small data

Marta Milia I Mappare la distruzione I DOI:10.12977/ocula2019-19

2013 "Le interfacce ludiche dei luoghi quotidiani. Strategie ludiche location-based per imparare a giocare con i confini tra il qui e l'altrove", Mediascapes journal, 2, 2013, pp. 28-44.

Bratton, Benjamin H.

2008 “iPhone City”, in: Digital Urbanism, AD: Architectural Design.

de Souza e Silva, Adriana; Sheller Mimi (a cura di)

2015 Mobility and Locative Media, Routledge, London, New York.

De Certeau, Michel

1990 L’invention du quotidien. I Arts de faire, Paris, Gallimard.

Eugeni, Ruggero

2016 La condizione postmediale, Brescia, La Scuola.

Flem, Lydia

2005 Come ho svuotato la casa dei miei genitori, Milano, Archinto.

Frith, Jordan

2015 Smartphones as Locative Media, Hoboken, Wiley-Blackwell.

Galloway, Anne; Ward, Matthew

2006 "Locative Media As Socialising And Spatializing Practice: Learning From Archaeology", in Drew Hemment (eds.), Locative Media Special, LEA, vol. 14, n. 3, 2006, <http://leoalmanac.org/journal/vol_14/lea_v14_no3-04/asant. html> (ultimo accesso 12 ottobre 2017).

Graham, Stephen; Marvin, Simon

2002 Città e comunicazione, Bologna, Baskerville.

La Cecla, Franco

1992 “Come stanno le cose”, in Borsari A. (a cura di), L'esperienza delle cose, Genova, Marietti.

Manovich, Lev

2013 "Media Visualization: Visual Techniques for Exploring Large Media Collections", Pdf reperibile all'indirizzo: < http://manovich.net/content/o4projects/o69-media-visualization-visual-techniques-for-exploring-largemedia-collections/66-article-2011.pdf> (ultimo accesso 20 settembre 2017).

Micalizzi, Alessandra

2012 "Un mondo di cose: scosse, oggetti e trauma", in Minardi E., Salvatore R. (a cura di), OR. eS. TE, Homeless Book, Teramo, (pp. 187-203).

Micalizzi, Alessandra; Farinosi, Manuela

2012 "L’Aquila 2.0: partecipazione dal basso nel primo disastro italiano dell'era digitale", in Minardi E., Salvatore, R. (a cura di), OR. eS. TE, Homeless Book, Teramo (pp. 157-176).

Montanari, Federico

2016 "Ancora un "turn"? La svolta Locative nei media e sue possibili implicazioni socio-semiotiche. Casi, esempi, e questioni.”, in Ferraro G., Lorusso A.M. (a cura di), Nuove forme di interazione. Dal Web al Mobile, Libellula, Lecce, pp. 159-174. 


\section{Dcula ${ }^{21}$}

Geosemiotica: dai locative media, alle immagini diffuse, ai big e small data

Marta Milia I Mappare la distruzione I DOI:10.12977/ocula2019-19

Pozzato, Maria Pia; Demaria, Cristina

2006 "Etnografia urbana: modi d'uso e pratiche dello spazio", in Pezzini I., Marrone G. (a cura di), Senso e metropoli, Roma, Meltemi, pp. 193-210

Prunesti, Alessandro; Casagrande, Gianluca

2010 "Geografia sociale, comunicazione localizzata e realtà aumentata”, in Cerreti C., Dumont I., Tabusi M. (a cura di), Geografia sociale e democrazia. La sfida della comunicazione, Roma, Aracne, pp.105-120

Thielmann, Tristan

2010 "Locative Media and Mediated Localities: An introduction to Media Geography”, in: Aether. The journal or media geography, Vol. V.A. 1-17, March, Northridge, California State University.

Vallega, Adalberto

2003 Geografia culturale. Luoghi, spazi, simboli, UTET, Bologna.

Wilken, Ronan; Goggin, Gerard (eds.)

2015 Locative Media, London and New York, Routledge.

Marta Milia (1986), nel 2017 ha conseguito il Dottorato di Ricerca in Semiotica e Media presso l'università degli studi di Torino; vincitrice di borsa Inps con un progetto sulla comunicazione social e in particolare sul linguaggio politico usato su Twitter e nuovi processi narrativi dell'informazione 2.0. Ha presentato la tesi "L'informazione in rete e i social media: per una semiotica della comunicazione 2.0”. Laureata in Linguistica Spagnola applicata al giornalismo e in Informazione e Editoria con una tesi dal titolo "Processo di notiziabilità e giornalismo di guerra: chi parla del Kurdistan?", ha collaborato con l'emittente televisiva ligure Primocanale. Svolge studi nell'ambito della comunicazione politica e dei media digitali e ha pubblicato articoli per riviste specializzate in comunicazione e semiotica tra le quali Lexia e Carte Semiotiche. 